\title{
Viaje al centro de la matriz cerámica: estudio sobre los usos de vasijas arqueológicas provenientes del sitio Tardío Loma l’Ántigo (valle del Cajón, Catamarca, Argentina) a partir del análisis químico de residuos culinarios
}

\author{
Sofía Fernández Sancha, Irene Lantos, María Fabiana Bugliani y Marta S. Maier \\ Recibido 09 de septiembre 2020. Aceptado 01 de diciembre 2020
}

\begin{abstract}
RESUMEN
En el presente trabajo se dan a conocer los resultados de estudios químicos de residuos lipídicos recuperados en las matrices cerámicas de contenedores del sitio arqueológico Loma l’Ántigo, un poblado Tardío del valle del Cajón (Catamarca, Argentina). Se analizaron los residuos de 12 muestras arqueológicas por medio de cromatografía gaseosa con detector de ionización de llama (GC-FID) y una olla etnográfica mediante GC-FID y cromatografía gaseosa acoplada a espectrometría de masa (GC-MS), con fines comparativos. La identificación de distintos ésteres metílicos de ácidos grasos (FAMEs) en nueve de las muestras arqueológicas y en la olla etnográfica resulta evidencia del empleo de los contenedores en distintas actividades culinarias, que implicaron la circulación de sustancias de origen vegetal y animal. En la mayoría de estos casos, se identificaron biomarcadores de grasas de rumiantes compatibles con camélidos. La articulación de los estudios químicos con otras fuentes de indagación permitió constatar el empleo de distintos tipos de contenedores en el almacenamiento, elaboración y servicio de comidas y/o bebidas.
\end{abstract}

Palabras clave: Residuos lipídicos; Cerámica; Alimentación; Período Tardío; Catamarca.

Journey to the center of the ceramic matrix: a study of the uses of archaeological vessels from the Late Loma l’Ántigo site, valle del Cajón, Catamarca, Argentina, through the chemical analysis of culinary residues

\begin{abstract}
In this paper, the results of chemical studies of lipid residues recovered from the ceramic matrices of containers from the archaeological site of Loma l'Antigo, a late settlement in the Valle del Cajón, Catamarca, Argentina, are presented. Residues extracted from 12 archaeological samples were analyzed by gas chromatography with flame ionization detection (GC-FID), and an ethnographic pot was studied by GC-FID and gas chromatography coupled to mass spectrometry (GC-MS) for comparative purposes. The identification of different fatty acid methyl esters (FAMEs) in nine of the archaeological samples and in the ethnographic

\footnotetext{
Sofía Fernández Sancha. Instituto de las Culturas (IDECU), Universidad de Buenos Aires-Consejo Nacional de Investigaciones Científicas y Técnicas (CONICET). Moreno 350 (CP I091). Ciudad Autónoma de Buenos Aires, Argentina. E-mail: sofifernandezsancha@gmail.com

Irene Lantos. IDECU, Universidad de Buenos Aires-CONICET. Moreno 350 (CP I09I), Ciudad Autónoma de Buenos Aires, Argentina. Unidad de Microanálisis y Métodos Físicos Aplicados a la Química Orgánica (UMYMFOR), CONICETDepartamento de Química Orgánica, Facultad de Ciencias Exactas y Naturales, Universidad de Buenos Aires. Intendente Güiraldes 2160 (1428), Ciudad Autónoma de Buenos Aires, Argentina. E-mail: irenelantos@qo.fcen.uba.ar

María Fabiana Bugliani. IDECU, Universidad de Buenos Aires-CONICET. Moreno 350 (CP I09I), Ciudad Autónoma de Buenos Aires, Argentina. E-mail: fbugliani@gmail.com

Marta Maier. UMYMFOR, CONICET-Departamento de Química Orgánica, Facultad de Ciencias Exactas y Naturales, Universidad de Buenos Aires. Intendente Güiraldes 2160 (1428), Ciudad Autónoma de Buenos Aires, Argentina. E-mail: maier@qo.fcen.uba.ar
}

Intersecciones en Antropología 22(1), enero-junio: 41-53. 2021. ISSN-e 1850-373X

https://doi.org/10.37176/iea.22.1.2021.583

Facultad de Ciencias Sociales - UNICEN - Argentina 
pot is evidence of the use of the containers in different culinary activities that involved the circulation of substances of plant and animal origin. Various samples showed the presence of ruminant fat biomarkers compatible with camelids. The articulation of chemical studies with other sources of inquiry allowed us to verify the use of different types of containers for storage, preparation and service of foods and/or drinks.

Keywords: Lipid residues; Ceramics; Feeding; Late period; Catamarca.

\section{INTRODUCCIÓN}

La alimentación del pasado ha sido abordada mediante diversas vías de investigación como la zooarqueología, la arqueobotánica, la etnohistoria, la química y la bioarqueología, entre otras. Entre las técnicas químicas, se destacan los análisis de residuos lipídicos hallados en piezas cerámicas (Sánchez Vizcaíno y Cañabate Guerrero, 1998; Evershed, 2008a; Colombini y Modugno, 2009; Regert, 2011). Este tipo de estudios se desarrollan desde hace varios años en la arqueología del noroeste argentino con el objeto de conocer algunos de los recursos utilizados en la elaboración de comidas y/o bebidas en el pasado prehispánico (Pazzarelli, 2011; Amuedo, 2012; Ortiz y Heit, 2013; Lantos et al., 2015).

La ventaja de investigar los residuos culinarios preservados en los contenedores cerámicos es que constituyen una evidencia de los usos que tuvieron a lo largo de sus historias de vida (Skibo, 2015;
Roffet-Salque et al., 2017). Los residuos invisibles absorbidos dentro de las matrices cerámicas pueden ser recuperados, ya que las porosidades actúan como trampas que los protegen de los factores de degradación ambiental. Los estudios experimentales han aportado la información de base necesaria para la interpretación de los perfiles lipídicos y la búsqueda de biomarcadores (Malainey, 1999; Eerkens, 2007; Frère, Costenla, Bayón y González, 2010; Lantos, Maier y Ratto, 2012).

En esta oportunidad, se estudiaron los residuos lipídicos de un conjunto cerámico recuperado en el sitio Loma I'Ántigo mediante cromatografía gaseosa (CG-FID) con el objetivo de conocer cuáles fueron los recursos procesados y consumidos, y a su vez, obtener información acerca de los distintos usos de los contenedores cerámicos.

Loma l'Ántigo se ubica en el sector medio del vaIle del Cajón, en la provincia de Catamarca (Figura 1), y se caracteriza por ser un poblado residencial.
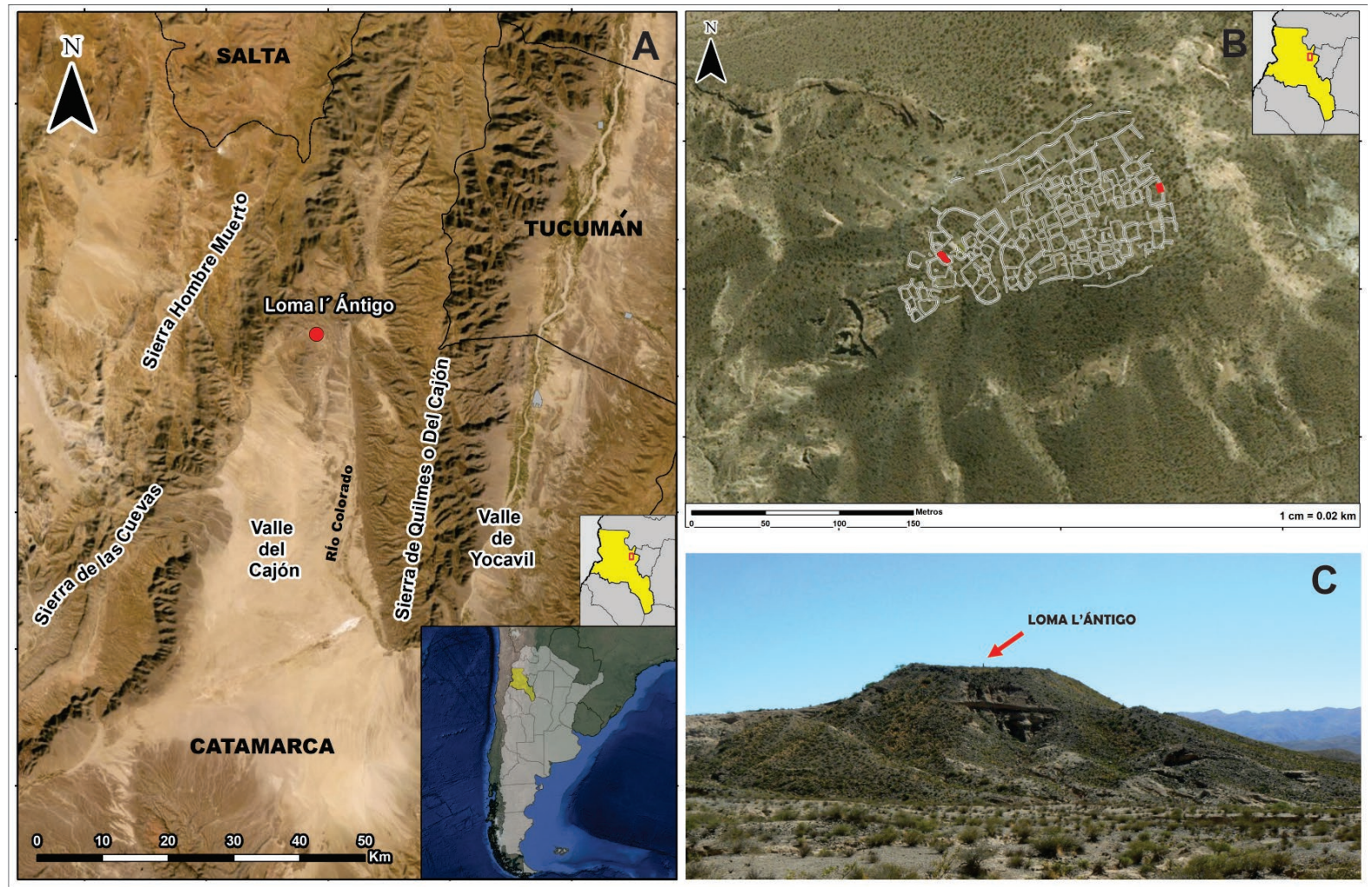

Figura 1. A) Mapa del área de estudio donde se observa la ubicación del sitio arqueológico Loma l’Ántigo (valle del Cajón, Catamarca); B) Plano arquitectónico del poblado sobre foto aérea de la loma; y C) Loma donde se emplaza el sitio. 
El sitio está asentado sobre la cima aplanada de una loma y se compone de 111 recintos de formas poligonales construidos con bloques de piedra, que ocupan 1,6 ha. Los nueve fechados radiocarbónicos obtenidos para el sitio muestran que estuvo habitado desde fines del siglo XIII hasta fines del siglo XV (Bugliani, 2018).

Los casos aquí analizados provienen de dos estructuras: la E25, situada en el extremo este del sitio, y la E93, en su sector oeste. La primera, de forma rectangular, fue excavada parcialmente en un área de $11 \mathrm{~m}^{2}$. La segunda, una habitación alargada de $45 \mathrm{~m}^{2}$, fue excavada en su totalidad. A partir del análisis de fragmentos cerámicos provenientes de ambas estructuras, se constató la presencia de conjuntos alfareros similares en los dos extremos del sitio (Figura 2).

El estudio de la estructura E25 mostró un área de combustión situada de manera adyacente a la pared noreste, asociada a diferentes recipientes cerámicos, restos óseos de fauna termoalterados y macrorrestos vegetales carbonizados. El análisis distribucional de los materiales recuperados en la estructura E93 permitió establecer la existencia de dos áreas de combustión y, asociadas a ellas, dos conjuntos materiales conformados por piezas cerámicas, desechos líticos, restos óseos de fauna y macrorrestos vegetales carbonizados (Bugliani, 2018). Se considera que en ambas estructuras se produjeron diferentes prácticas domésticas, entre las que se incluyen actividades de combustión, consumo y descarte de restos de fauna, como así también el procesamiento y consumo de plantas alimenticias. Las muestras de cerámica seleccionadas proceden de las áreas de actividad mencionadas, y a partir de su estudio químico se pretende recabar información que permita evaluar el empleo de estas vasijas en la elaboración de comidas y/o bebidas.

\section{MATERIALES}

Para investigar los usos de los contenedores cerámicos de las estructuras E93 y E25 mediante análisis químicos de residuos orgánicos se seleccionaron 12 muestras (Tabla 1). Estas son representativas del repertorio de formas y tipos cerámicos reconocidos en el sitio. Las siluetas de las piezas fueron reconstruidas con anterioridad mediante el número mínimo de vasijas (NMV). Siete muestras corresponden a piezas distintas reconstruidas de la estructura E93 y fueron denominadas M4, M5, M6, M7, M9, M10 y M11. Otras cinco proceden de la estructura E25. Las muestras M2, M3 y M12 corresponden a piezas de formas diferentes, mientras que

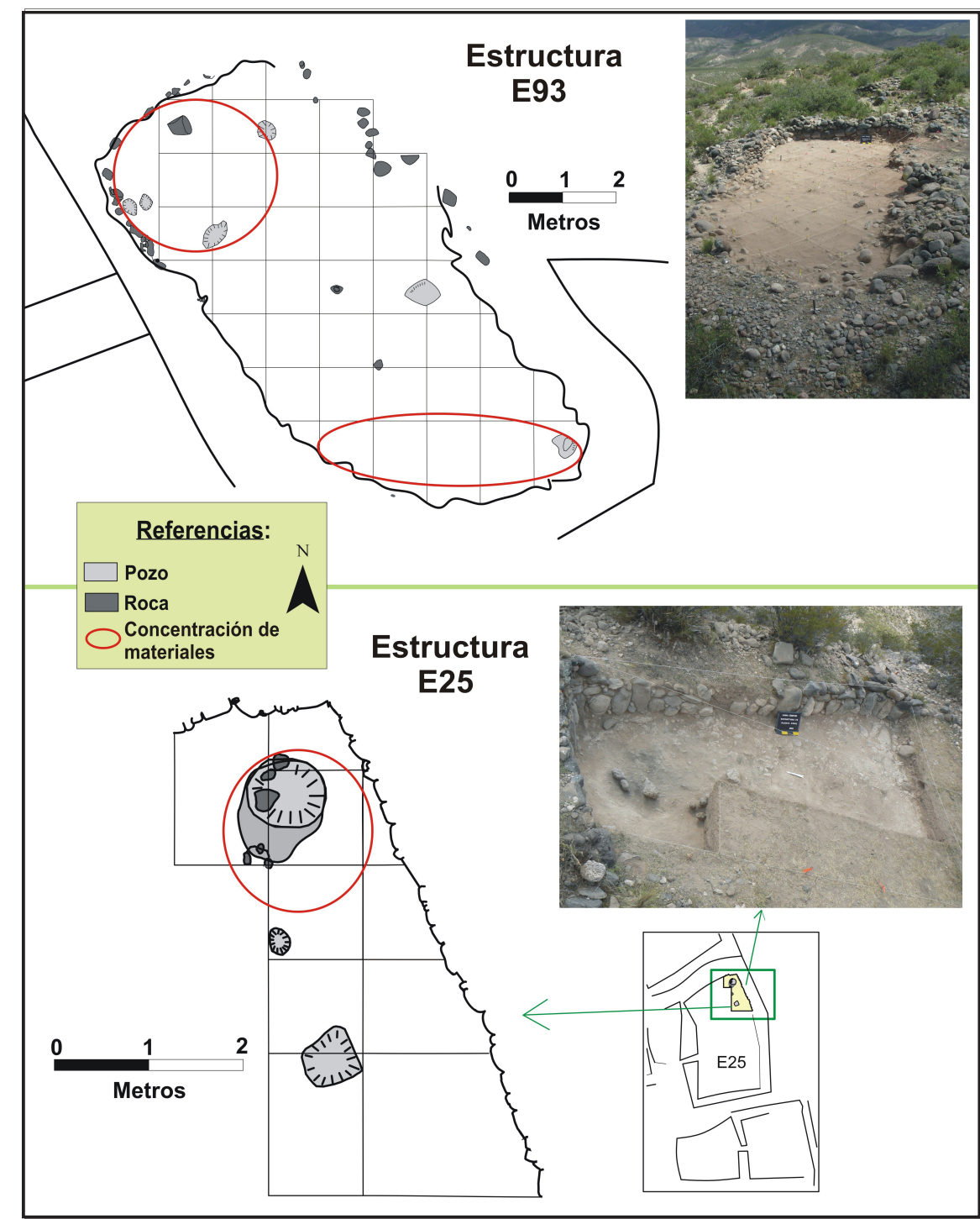

Figura 2. Estructuras E93 y E25 de donde provienen los materiales arqueológicos analizados. 
las dos restantes -denominadas M1 y M8- representan sectores de una misma vasija (borde y base de una pieza grande); ambas fueron analizadas en búsqueda de posibles diferencias en los residuos de cada zona (Figura 3).

Las muestras M1, M4, M5, M8 y M12 pertenecen a ollas globulares de tipo Ordinario Alisado y Peinado de tamaños grandes. Las muestras M3, M7, M10 y M11 corresponden a piezas del tipo Santamariano Bicolor. Tres son cuencos de cuello corto evertido (M7, M10 y M11). Las paredes exteriores presentan un baño blanco o diseños pintados con color negro sobre el baño blanco. Las paredes internas poseen diseños en negro pintados sobre la pasta de color naranja. La M3 corresponde al cuerpo de una vasija de tipo Santamariano Bicolor con aplique de cabeza antropomorfa, posiblemente perteneciente a una pieza en forma de urna (fragmento C1274-T1). La M9 (fragmento C1088-2) pertenece a una pieza pintada en Negro sobre Rojo y consiste en el cuerpo de una vasija de terminación alisa-
(M2), NMV4 (M6) y el C1278-T6 (M12). Estos ejemplares poseen un punto de inflexión muy destacado en el diámetro máximo, cuello evertido, superficies alisadas, y generalmente tienen mamelones o apliques circulares con una cruz excisa adheridos al cuerpo. Son de menor tamaño, paredes más delgadas y presentan bases más angostas que la variante anterior. Por último, la variante $\mathrm{C}$, formada por el NMV2/3 (M5), presenta boca ancha, borde directo y no tiene cuello. La superficie presenta zonas de terminación alisada y peinada y posee un asa de tipo otomorfa adherida muy cerca del borde. Las ollas pertenecientes a la variante A habrían sido empleadas para el almacenamiento de diferentes sustancias, mientras que las variantes B y C habrían sido utilizadas en la cocción de alimentos.

Además, se incorporó una muestra de referencia proveniente del raspado del interior de una olla de cerámica actual perteneciente a una pobladora de la localidad de La Quebrada (valle del Cajón). Esta olla fue utilizada por su dueña y anteriormente por

da con diseños geométricos.

Con antelación, el estudio del conjunto cerámico hallado en Loma I'Ántigo nos permitió diferenciar tres variantes $(\mathrm{A}$, B y C) dentro de las ollas globulares con bases bicóncavas en forma de pie o pedestal. La variante A está constituida por los NMV1 (M4) y NMV251 (M1 y M8) de tamaños grandes, cuello corto, bordes evertidos, de coloración gris y terminación de superficie peinada. La B está compuesta por Ios NMV252

\begin{tabular}{|c|c|c|c|c|c|c|c|}
\hline Muestra & Código & Estr. & Forma & Estilo & Sector vasija & FAMEs & Rastro uso \\
\hline M1 & $\begin{array}{l}\text { C853- T6/ } \\
\text { NMV251 }\end{array}$ & 25 & $\begin{array}{c}\text { Olla globular } \\
\text { con pie }\end{array}$ & $\begin{array}{l}\text { Ordinario } \\
\text { Peinado }\end{array}$ & Base/cuerpo & No & $\begin{array}{l}\text { Sup. int. } \\
\text { "picada" }\end{array}$ \\
\hline M2 & $\begin{array}{l}\text { C1290-T6/ } \\
\text { NMV252 }\end{array}$ & 25 & $\begin{array}{c}\text { Olla punto } \\
\text { angular } \\
\text { destacado }\end{array}$ & $\begin{array}{l}\text { Ordinario } \\
\text { Alisado }\end{array}$ & Cuerpo & No & $\begin{array}{l}\text { Sup. ext. } \\
\text { ennegrecida }\end{array}$ \\
\hline M3 & C1274-T1 & 25 & $\begin{array}{l}\text { Posible } \\
\text { tinaja/urna }\end{array}$ & $\begin{array}{c}\text { Santamariano } \\
\text { Bicolor }\end{array}$ & Cuerpo & Sí & - \\
\hline M4 & $\begin{array}{c}\text { C1127-T4/ } \\
\text { NMV1 }\end{array}$ & 93 & $\begin{array}{c}\text { Olla globular } \\
\text { con pie }\end{array}$ & $\begin{array}{l}\text { Ordinario } \\
\text { Peinado }\end{array}$ & Base/cuerpo & Sí & $\begin{array}{l}\text { Sup. int. } \\
\text { ennegrecida }\end{array}$ \\
\hline M5 & $\begin{array}{l}\text { C1098-T2/ } \\
\text { NMV2/3 }\end{array}$ & 93 & $\begin{array}{c}\text { Olla globular } \\
\text { sin cuello }\end{array}$ & $\begin{array}{l}\text { Ordinario Ali- } \\
\text { sado y Peinado }\end{array}$ & Borde & Sí & $\begin{array}{l}\text { Sup. ext. } \\
\text { ennegrecida }\end{array}$ \\
\hline M6 & $\begin{array}{l}\text { C1152-T4/ } \\
\text { NMV4 }\end{array}$ & 93 & $\begin{array}{c}\text { Olla punto } \\
\text { angular } \\
\text { destacado }\end{array}$ & $\begin{array}{l}\text { Ordinario } \\
\text { Alisado }\end{array}$ & Cuerpo & Sí & $\begin{array}{l}\text { Sup. ext. } \\
\text { ennegrecida }\end{array}$ \\
\hline M7 & $\begin{array}{l}\text { C1126-T9/ } \\
\text { NMV8 }\end{array}$ & 93 & $\begin{array}{l}\text { Cuenco con } \\
\text { cuello corto }\end{array}$ & $\begin{array}{c}\text { Santamariano } \\
\text { Bicolor }\end{array}$ & Cuerpo & Sí & - \\
\hline M8 & $\begin{array}{c}\text { C1280- } \\
\text { T11/ } \\
\text { NMV251 }\end{array}$ & 25 & $\begin{array}{l}\text { Olla globular } \\
\text { con pie }\end{array}$ & $\begin{array}{l}\text { Ordinario } \\
\text { Peinado }\end{array}$ & Borde & No & $\begin{array}{l}\text { Sup. int. } \\
\text { "picada" }\end{array}$ \\
\hline M9 & C1088-2 & 93 & $\begin{array}{c}\text { Forma } \\
\text { cerrada }\end{array}$ & $\begin{array}{c}\text { Pintado Negro } \\
\text { sobre Rojo } \\
\end{array}$ & Cuerpo & Sí & - \\
\hline M10 & $\begin{array}{c}\text { C1107-T2/ } \\
\text { NMV5 }\end{array}$ & 93 & $\begin{array}{l}\text { Cuenco con } \\
\text { cuello corto }\end{array}$ & $\begin{array}{c}\text { Santamariano } \\
\text { Bicolor }\end{array}$ & Base & Sí & - \\
\hline M11 & $\begin{array}{l}\text { C829-T5/ } \\
\text { NMV9 }\end{array}$ & 93 & $\begin{array}{l}\text { Cuenco con } \\
\text { cuello corto }\end{array}$ & $\begin{array}{c}\text { Santamariano } \\
\text { Bicolor }\end{array}$ & Cuerpo & Sí & - \\
\hline M12 & C1278-T6 & 25 & $\begin{array}{c}\text { Olla punto } \\
\text { angular } \\
\text { destacado }\end{array}$ & $\begin{array}{l}\text { Ordinario } \\
\text { Alisado }\end{array}$ & Cuerpo & Sí & - \\
\hline M13 & $\begin{array}{c}\text { Olla } \\
\text { etnográfica }\end{array}$ & Dora & Olla globular & $\begin{array}{l}\text { Ordinario } \\
\text { Alisado }\end{array}$ & Base/borde & Sí & $\begin{array}{l}\text { Sup. int. y } \\
\text { ext. enne- } \\
\text { grecidas }\end{array}$ \\
\hline
\end{tabular}

Tabla 1. Descripción de las muestras cerámicas analizadas. 
su madre para preparar maíz tostado, una receta típica de la zona que consiste en el tostado de los granos de maíz junto con cenizas mientras se lo va removiendo a fuego lento hasta que la cáscara se tuesta. Se le asignó el nombre M13 y se trata de una olla de cuerpo globular, de base plana y destacada, con un cuello muy corto evertido y una abertura de boca oblonga de unos 300 mm en su lado más largo. La terminación de superficie externa es alisada y se encuentra cubierta de hollín (Figura 3).

\section{METODOLOGÍA}

Se llevó a cabo la extracción de los lípidos de las 12 muestras arqueológicas y una etnográfica. En el primer caso, se cortaron fragmentos de aproximadamente 5 g y se eliminaron las superfi-

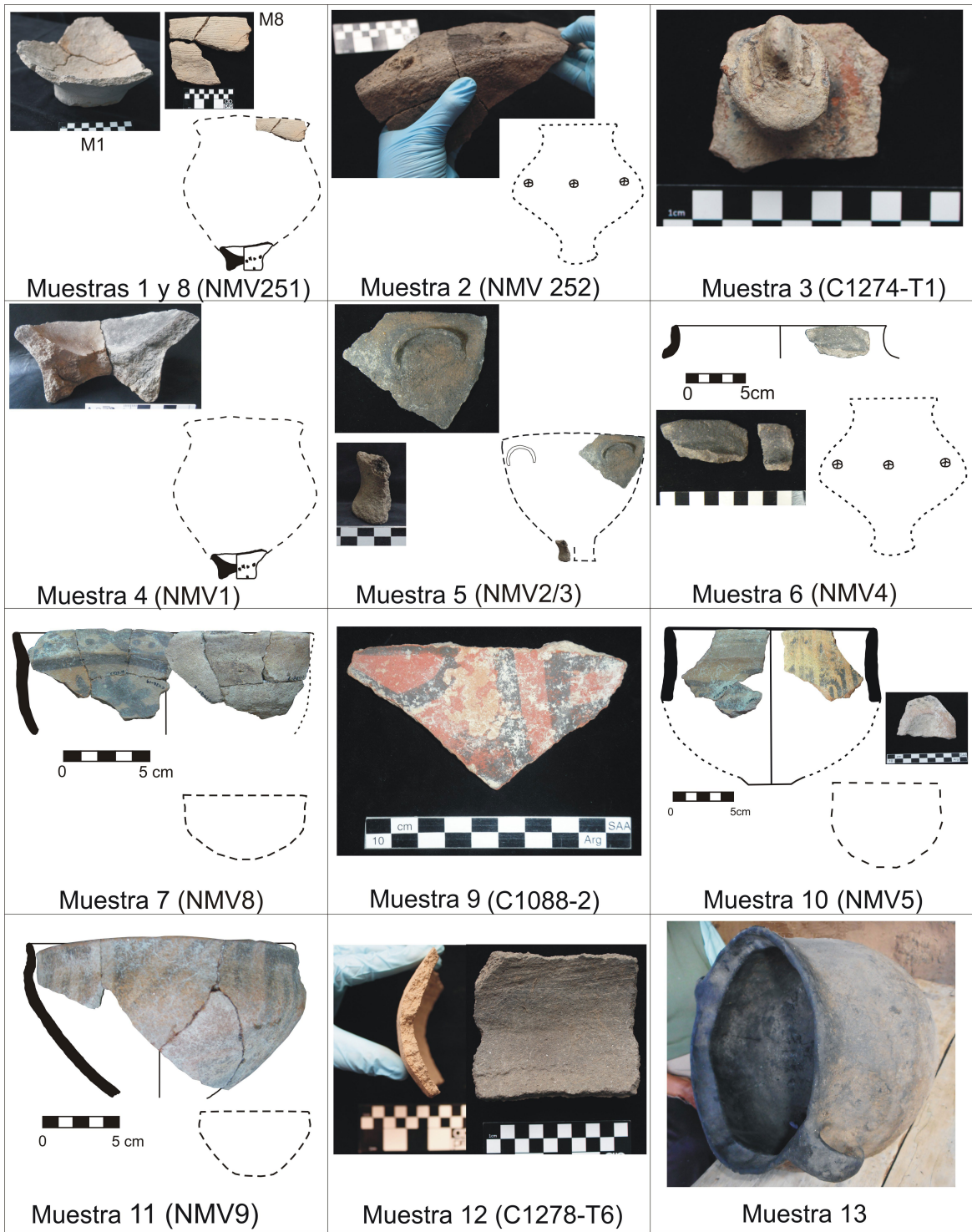

Figura 3. Ollas cerámicas de donde provienen las muestras.

cies externas e internas con un taladro de punta amoladora. Luego, cada muestra se molió en un mortero de porcelana. En el segundo caso se trabajó con 0,017 g del material raspado del interior de la vasija etnográfica.

Se trataron $5 \mathrm{~g}$ de muestra con $25 \mathrm{ml}$ de una mezcla de cloroformo: metanol en proporción 2:1 (dos veces) (Folch, Lees y Sloane Stanley, 1957), en un baño de ultrasonido durante 15 minutos. Se pasó la mezcla a tubos y se centrifugó a 2000 rpm durante 5 minutos para separar los restos de cerámica molida y recuperar el líquido sobrenadante. Se filtró el sobrenadante empleando papel de filtro en una ampolla, se agregaron $16 \mathrm{ml}$ de agua destilada y se agitó. Se recuperó la fase orgánica que contenía el extracto lipídico total y se evaporaron las muestras utilizando una suave corriente de nitrógeno. Se pesó el extracto y se reservó a $-18{ }^{\circ} \mathrm{C}$.
Se tomó una alícuota de $5 \mathrm{mg}$ del extracto lipídico total y se le realizó un tratamiento químico para la obtención de los derivados ésteres metílicos de ácidos grasos (FAMEs, por sus siglas en inglés), siguiendo el procedimiento detallado en Lantos et al. (2020).

Las muestras fueron analizadas por cromatografía gaseosa (GC-FID) en un Focus GC (Thermo Finnigan Corporation), siguiendo el procedimiento detallado en Lantos et al. (2020). Los FAMEs se identificaron por sus tiempos de retención por comparación con un estándar comercial (FAME C4C24 Mix, Supelco). Las abundancias relativas de los FAMEs fueron obtenidas a partir de las áreas debajo de cada pico, y fueron expresadas como porcentajes del área total de los FAMEs (Figura 4, Tabla 2).

La muestra M13 fue además analizada por cromatografía gaseosa acoplada a espectrometría de 


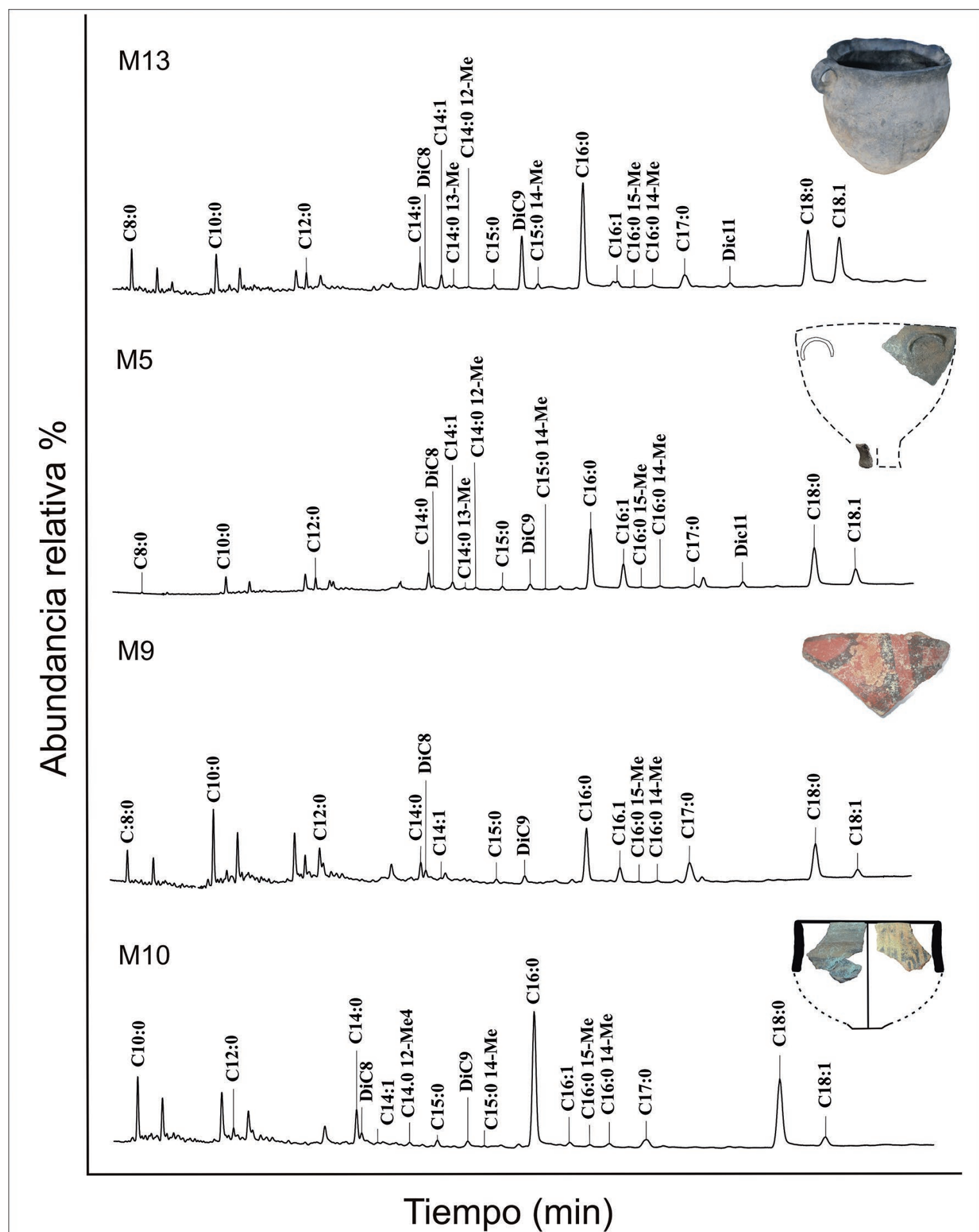

Figura 4. Cromatogramas de ésteres metílicos de ácidos grasos (FAMEs) de tres muestras arqueológicas (M 9, M5 y M10) y de la olla etnográfica (M13). Solo se muestran algunos de los contenedores arqueológicos a modo de ejemplo, debido a una cuestión de espacio.

masa (GC-MS) en un equipo Shimadzu GCMS QP5050A (Kyoto, Japón), equipado con una columna capilar ZB5 (Phenomex, 5\% fenil-95\% dimetilpolisiloxano, $30 \mathrm{~m}$ de largo, 0,25 $\mathrm{ml}$ de diámetro interno, 0,25 $\mu \mathrm{m}$ de espesor del film). El gas carrier fue helio $(0,9 \mathrm{ml} / \mathrm{min}$ de flujo continuo). La inyección manual fue en modo split a $250{ }^{\circ} \mathrm{C}$. La rampa de temperatura fue: $110^{\circ} \mathrm{C}, 10^{\circ} \mathrm{C} / \mathrm{min}$ hasta $280{ }^{\circ} \mathrm{C}$ (45 min). El espectrómetro de masa fue operado en modo de impacto electrónico a 70 eV. La temperatura de la fuente de ionización fue de $280{ }^{\circ} \mathrm{C}$. La identificación de los compuestos fue realizada por comparación de los tiempos de retención de los FAMEs con estándares comerciales (FAME C4-C24 
Mix [Supelco]; Dimethyl azelate, Dimethyl suberate [Sigma-Aldrich]) y por los patrones de fragmentación en los espectros de masa que se compararon con librerías de espectros. El análisis por GC-MS de la muestra M13 nos permitió confirmar la identidad de los picos e identificar compuestos minoritarios para los cuales no hay estándar comercial.

\section{RESULTADOS}

Los resultados de los análisis químicos mostraron que 9 de los 12 casos arqueológicos (M3, M4, M5, M6, M7, M9, M10, M11 y M12), junto con la olla etnográfica (M13), presentaron residuos lipídicos como producto del contacto con ingredientes de origen animal y/o vegetal. La concentración de lípidos por gramo de matriz cerámica en las muestras varió de $32 \mu \mathrm{g}$ a $1073 \mu \mathrm{g}$. En el caso de la olla etnográfica se observó una concentración de $508.187 \mu \mathrm{g}$ de lípidos por gramo de residuo raspado (Tabla 2). Las concentraciones observadas en las muestras arqueológicas se condicen con los valores habituales hallados en cerámicas arqueológicas con residuos producto de actividades antrópicas (Evershed, 2008b). En las muestras M1, M2 y M8 no se detectaron FAMEs.

A partir de los análisis cromatográficos se identificaron FAMEs en el rango de C8 a C18 en las diferentes muestras. Los ácidos grasos saturados identificados fueron caprílico (C8:0), cáprico (C10:0), láurico (C12:0), mirístico (C14:0), pentadecanoico (C15:0), palmítico (16:0), margárico (C17:0) y esteárico (C18:0). A su vez, se encontraron los ácidos grasos insaturados miristoleico (C14:1), palmitoleico (16:1) y oleico (C18:1).

También se detectaron en la mayoría de las muestras analizadas ácidos dicarboxílicos como el subérico (diC8) y el azelaico (diC9). Estos compuestos son considerados productos de degradación de ácidos grasos insaturados y se podrían asociar con la presencia de lípidos vegetales (Dunne et al., 2016).

Además, en varias muestras se observaron ácidos grasos ramificados de 15, 16 y 17 carbonos: C14:0 13-Me, C14:0 12-Me, C15:0 14-Me, C16:0 15-Me y C16:0 14-Me (Tabla 2).

Los ácidos grasos saturados de cadena impar (C15) y (C17), tanto lineales como ramificados, son considerados biomarcadores de la grasa de animales rumiantes debido al proceso metabólico bacteriano acaecido dentro del rumen (Dudd, Regert y Evershed, 1998; Martínez Marín et al., 2010). Aunque pequeñas proporciones de estos compuestos también podrían estar ligados a un

\begin{tabular}{|c|c|c|c|c|c|c|c|c|c|c|}
\hline FAMEs & M3 & M4 & M5 & M6 & M7 & M9 & M10 & M11 & M12 & M13 \\
\hline $\begin{array}{l}\text { Concentración de } \\
\text { lípidos }(\mu \mathrm{g} / \mathrm{g})\end{array}$ & $\mathrm{s} / \mathrm{d}$ & 113 & 58 & 478 & 32 & 643 & 138 & 1073 & 138 & 508187 \\
\hline C8:0 & - & - & 0,2 & - & 0,4 & 5,1 & - & - & 1,5 & 3,9 \\
\hline C10:0 & 4,5 & 1,1 & 3,3 & 6,6 & 6,5 & 14,1 & 7,7 & 6,8 & 9,5 & 4,8 \\
\hline C12:0 & 3,3 & 3,5 & 2,2 & - & 1,5 & 3,5 & 1,2 & - & 8,9 & 1,5 \\
\hline C14:0 & 5,3 & 5,3 & 4,4 & 3,3 & 9,6 & 4,3 & 6,2 & 3,4 & 2,4 & 3,8 \\
\hline $\operatorname{dic} 8$ & 3,2 & 1,3 & 0,4 & 3,0 & 1,4 & 2,0 & 1,7 & 1,3 & 1,9 & 0,2 \\
\hline C14:1 & - & - & 1,8 & 5,7 & 0,4 & 0,2 & 0,2 & - & 1,0 & 2,2 \\
\hline C14:0 13-Me & - & - & 0,5 & - & - & - & - & - & 0,5 & 0,4 \\
\hline C14:0 12-Me & - & - & 0,7 & - & - & - & 0,7 & - & 0,3 & 0,2 \\
\hline C15:0 & 1,4 & 1,0 & 1,1 & 3,1 & 3,3 & 1,1 & 1,4 & 1,9 & 0,8 & 0,8 \\
\hline $\operatorname{dic} 9$ & 7,6 & 3,6 & 2,6 & 6,1 & 2,0 & 2,7 & 1,2 & 2,4 & 3,2 & 10,2 \\
\hline C15:0 14-Me & - & - & 0,2 & - & - & - & 0,2 & - & - & 0,9 \\
\hline C16:0 & 36,3 & 12,0 & 28,3 & 34,9 & 25,3 & 21,8 & 42,0 & 40,6 & 28,3 & 26,8 \\
\hline C16:1 & 3,1 & - & 12,1 & 9,8 & 15,2 & 6,3 & 1,1 & - & 5,2 & 2,2 \\
\hline C16:0 15-Me & 5,3 & - & 0,4 & - & - & 0,3 & 0,6 & - & 0,7 & 0,2 \\
\hline C16:0 14-Me & - & - & 1,0 & - & - & 1,0 & 1,2 & - & 0,4 & 1,0 \\
\hline C17:0 & 3,1 & 2,1 & 1,7 & 1,0 & 13,3 & 12,4 & 4,1 & 6,7 & 3,4 & 5,0 \\
\hline diC11 & - & - & 2,2 & - & - & - & - & - & 0,7 & 1,1 \\
\hline C18:0 & 26,9 & 62,8 & 25,9 & 18,1 & 7,7 & 20,4 & 27,8 & 34,3 & 22,1 & 18,0 \\
\hline C18:1 & - & 7,3 & 10,9 & 8,5 & 13,4 & 4,8 & 2,7 & 2,7 & 9,1 & 17,0 \\
\hline \multicolumn{11}{|l|}{ ÍNDICES } \\
\hline $\mathrm{P} / \mathrm{E}$ & 1,3 & 0,2 & 1,1 & 1,9 & 3,3 & 1,1 & 1,5 & 1,2 & 1,3 & 1,5 \\
\hline I/P & 0,1 & 0,0 & 0,1 & 0,1 & 0,3 & 0,2 & 0,1 & 0,1 & 0,1 & 0,1 \\
\hline
\end{tabular}

Tabla 2. Abundancia relativa de los FAMEs identificados en cada muestra e índices P/E e I/P. 
origen microbiano posdepositacional, su presencia transversal y abundante indicaría que se trata de lípidos de origen rumiante. Biomarcadores idénticos a estos fueron detectados en lípidos residuales en materiales arqueológicos, cuyo origen fue asociado a la grasa de camélidos sudamericanos (Maier et al., 2007; Vázquez et al., 2008; Lantos et al., 2015; Miyano et al., 2017). Los resultados obtenidos concuerdan con la información zooarqueológica obtenida en los contextos de Loma l'Ántigo de donde provienen las muestras. Son preponderantes los restos pertenecientes a la familia Camelidae, que incluyen elementos anatómicos identificados como Lama sp. y otros que podrían pertenecer a Vicugna vicugna. Además, se identificaron marcas de procesamiento en ejemplares del subconjunto Camelidae (Belotti López de Medina y Bugliani, 2019).

El índice P/E (relación entre los porcentajes de los ésteres metílicos de los ácidos C16:0 y C18:0) es ampliamente utilizado para reconocer el origen vegetal o animal de las sustancias grasas antiguas (Eerkens, 2005; Regert, 2011). Los valores entre 1 y 3 son considerados indicadores del aporte de grasas animales, mientras que aquellos mayores a 3 son evaluados como indicadores del aporte de aceites vegetales (Lantos et al., 2015). En la Tabla 2 se muestra el cálculo de este índice en las muestras estudiadas. De manera complementaria se calcularon las relaciones entre la suma de los porcentajes de los FAMEs saturados de cadena impar $(\mathrm{C} 15+\mathrm{C} 17$, tanto lineales como ramificados) y la suma de los saturados de cadena par (C8:0 + C1 $0: 0+\mathrm{C} 12: 0+\mathrm{C} 14: 0+\mathrm{C} 16: 0+\mathrm{C} 18: 0)(\mathrm{I} / \mathrm{P})$, para indagar sobre la presencia o ausencia de grasas de animales rumiantes. Los valores por encima de 0,04

\section{Relación entre índices de ácidos grasos}

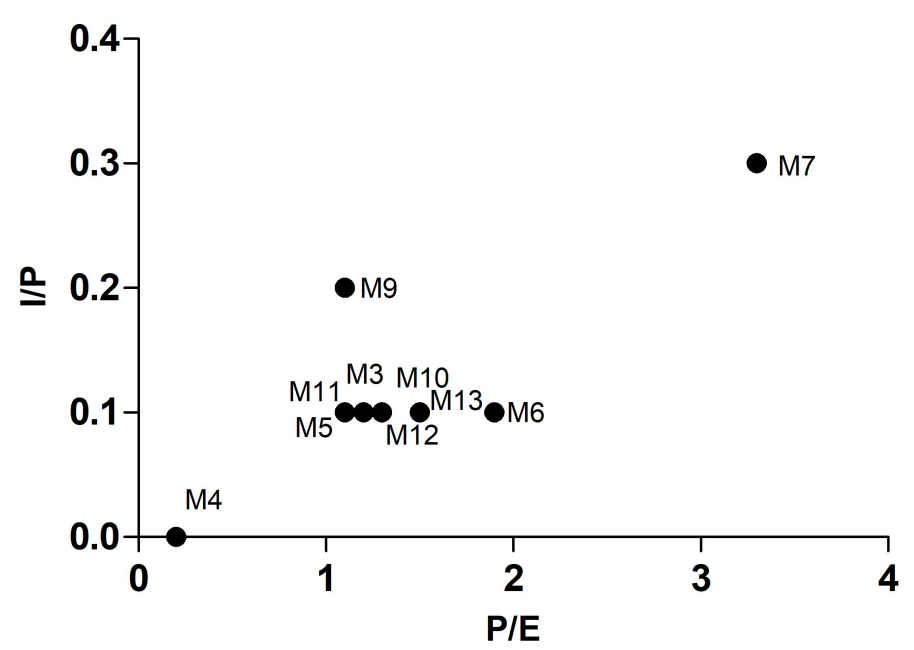

Figura 5. Relación entre los índices P/E y I/P. son indicadores de la presencia de tales grasas sensu Malainey (1997) y Eerkens (2005), (ver Tabla 2).

Los valores $\mathrm{P} / \mathrm{E}$ variaron en torno a una media de 1,4 $\pm 0,8$, lo que evidencia una fuerte presencia de grasa animal en la mayoría de las muestras. Sin embargo, algunas tuvieron valores superiores a la media y esto sugiere posibles mezclas que también incluyeran un componente vegetal. Por otra parte, los valores del índice I/P variaron entre 0,1 y 0,3 , lo cual refuerza la hipótesis del origen rumiante de las sustancias lipídicas en las muestras.

La Figura 5 muestra cómo se relacionan ambos índices, y permite observar un agrupamiento central de la mayoría de las muestras arqueológicas (M3, M5, M6, M9, M10, M11 y M12) junto con la muestra de referencia etnográfica M13 y la presencia de dos casos aislados (M4 y M7) cuyos valores son disímiles a los del grupo central.

Las muestras que se agrupan en el centro tienen valores del índice P/E típicos de grasa animal, mientras que el índice I/P señala el origen rumiante de estos lípidos. En la olla etnográfica (M13) están presentes la totalidad de los compuestos identificados en el conjunto de las muestras analizadas. El valor del índice P/E $(1,5)$ establece un origen animal de las grasas. A su vez, el índice I/P es mayor a 0,04 y posee biomarcadores de rumiantes. Por otro lado, se identificaron tres ácidos dicarboxílicos, incluyendo un porcentaje importante de azelaico (Tabla 2). Esto último sugiere un aporte vegetal a la mezcla. Con respecto a las ollas arqueológicas de este grupo, observamos distintos resultados. Las muestras M3, M5, M6, M9, M10, M11 y M12 poseen valores de índice P/E entre 1 y 2, que las ubican dentro del rango de las grasas de origen animal. Estas procederían de animales rumiantes, si se tiene en cuenta también el índice I/P mayor a 0,04 en todos los casos. A su vez, dentro de este conjunto, los ejemplares M3, M5, M9, M10 y M12 presentan ácidos grasos impares ramificados que son biomarcadores de rumiantes.

Por su parte, la muestra M7 posee un valor de índice P/E $(3,3)$ que sugiere una mezcla con una importante proporción de aceites vegetales, así como también el aporte menor de grasas animales. La abundancia de C17:0 en ausencia de otros biomarcadores de rumiante podría adjudicarse a contaminación microbiana y explicaría el valor de 0,3 para el índice 
I/P. El origen principalmente vegetal del residuo lipídico es apoyado además por una supervivencia relativamente alta de ácidos grasos insaturados y ácidos dicarboxílicos.

En el caso de la muestra M4, se observan resultados particulares vinculados potencialmente a alteraciones que habría sufrido la pieza, ya que el sector estudiado de la vasija se encontraba tiznado en la superficie interna, mientras que otro fragmento del contenedor no lo estaba, lo que sugiere que el tizne fue un evento posdepositacional. El valor del índice P/E es tan bajo $(0,2)$ que no se puede estimar la presencia de componentes animales o vegetales. Es la única muestra en la que el índice para detectar la presencia de grasas de animales rumiantes dio en el límite de 0,04 y a su vez no posee biomarcadores de camélidos sudamericanos.

\section{DISCUSIÓN Y CONSIDERACIONES FINALES}

La interpretación sobre los usos que habrían tenido los contenedores cerámicos de Loma I'Ántigo estudiados surge de la articulación de los análisis químicos realizados con otras fuentes de indagación. Estas últimas incluyen características morfotecnológicas de las piezas, rastros visibles de uso y/o desgaste en las superficies de los contenedores, como así también información de índole contextual.

Como fue mencionado anteriormente, considerábamos que las ollas pertenecientes a la variante A podrían haber sido empleadas en el almacenamiento de distintas sustancias sin entrar en contacto con el fuego, y que las ollas constituyentes de las variantes B y C se habrían utilizado en la cocción diaria de alimentos, por lo que habrían sido expuestas al fuego de manera recurrente. Es así como los análisis químicos de los recipientes pertenecientes a estos dos grupos aportan información complementaria que sustenta las hipótesis previas.

De las tres piezas pertenecientes a la variante de ollas de contorno muy inflexionado y pie destacado se obtuvieron diferentes resultados. El NMV4 (M6) estuvo vinculado con la cocción de comidas que incluyeron animales rumiantes como los camélidos. Los ejemplares NMV4 y NMV252 (M2) poseen paredes externas ennegrecidas por la exposición al fuego, por lo que esperábamos encontrar restos de lípidos. Se ha demostrado que las ollas destinadas a la cocción de alimentos contienen mayor cantidad de lípidos extraíbles que aquellas empleadas en contenedores con funciones de almacenamiento (Kimpe et al., 2004). A su vez, las paredes delgadas, la inflexión en la zona del cuello y la abertura restringida reducen la evaporación y previenen la pérdida de calor, lo que, de acuerdo con datos etnográficos, las hace adecuadas para el hervido a fuego lento usualmente asociado con la preparación de carnes (Eerkens, 2005). Si bien el NMV252 no dio resultados positivos, no descartamos que haya contenido lípidos en el pasado y que estos se hayan degradado con el tiempo. Esta idea se refuerza por el hecho de que la olla se encontró asociada a una zona de combustión con restos de fauna y de distintas plantas con propiedades alimenticias.

Los dos ejemplares de las ollas de contorno inflexionado y cuello corto denominadas NMV1 (M4) y NMV251 (M1 y M8) presentan resultados disímiles. En la olla NMV251 no se han encontraron ácidos grasos en el interior de su matriz cerámica; este hecho, sumado a la ausencia de rastros de exposición al fuego en la pieza nos hace considerar que no fue empleada en la cocción de comidas y/o bebidas. Por otro lado, la superficie interna de la base se encuentra "picada" y recuerda alteraciones de uso vinculadas con la fermentación (Arthur, 2002; Skibo, 2015). Es por lo expuesto que no se descarta la posibilidad de que haya estado en contacto con sustancias alimenticias que no se hayan preservado a lo largo del tiempo por degradación. Si bien no tenemos resultados concluyentes para el perfil lipídico de la olla NMV1 (M4), este puede ser producto de una alteración y/o degradación de los residuos. La pieza fue encontrada rota por la mitad y uno de los dos fragmentos posee la superficie interna ennegrecida; sin embargo, consideramos que la alteración del color se produjo de manera posterior a su uso. Estos datos, junto con el hecho de que las vasijas son de tamaños grandes, con bases estables y paredes gruesas, nos hacen pensar que estas estuvieron destinadas al almacenamiento de líquidos u otras sustancias de bajos contenidos lipídicos.

En la olla NMV2/3 (M5) se encontraron restos de grasas de origen animal, particularmente de camélidos. Posee las paredes externas ennegrecidas por hollín; se encontró asociada a un fogón en cubeta y a la zona de mayor concentración de restos arqueológicos de la estructura E93, entre los cuales se incluyen restos zooarqueológicos. Por estos resultados, consideramos que la olla fue utilizada para cocinar alimentos de origen animal rumiante como los camélidos. 
Teniendo en cuenta los resultados químicos para los cuencos pintados del tipo Santamariano Bicolor (M7, M10 y M11) y considerando que estos no presentan rastros de haber sido expuestos al fuego, proponemos que no estuvieron involucrados en la cocción de alimentos, sino en el servicio de comidas o bebidas de origen vegetal y animal (rumiante). Esta interpretación se sustenta en el hecho de que las formas abiertas de estas piezas, con paredes delgadas y tamaños pequeños, son útiles para trasladar y servir distintas sustancias. La asociación de cuencos de estilo Santamariano con funciones culinarias semejantes a las planteadas aquí también se registró, por medio de análisis químicos, en ejemplares arqueológicos del valle de Yocavil provenientes de los sitios Fuerte Quemado Intihuatana y El Colorado (Lantos et al., 2018; Lantos et al., 2020).

En los contenedores pintados de formas cerradas representados por las muestras M3 y M9 también se detectó la presencia de grasas de origen animal rumiante y biomarcadores de camélidos. Al no haber encontrado restos de hollín en las superficies de estos fragmentos, creemos que no fueron expuestos al fuego para cocinar, pero sí que estuvieron involucrados en actividades culinarias como las de almacenamiento o servicio. Con respecto a la M3, se conoce que los apliques de cabezas antropomorfas por lo general forman parte de urnas santamarianas (Nastri, 2008), y existen trabajos sobre este tipo de piezas que han encontrado residuos lipídicos vinculados a posibles actividades de almacenamiento, preparación y servicio de líquidos o guisos en sitios de los valles Yocavil y Calchaquí Norte (Amuedo, 2012; Lantos et al., 2018; Lantos et al., 2020). Estos hallazgos resultan interesantes ya que exploran alternativas a la interpretación tradicional del empleo de las tinajas santamarianas asociadas principalmente con prácticas funerarias, como lo es su empleo en contextos domésticos (Greco, Marchegiani y Palamarczuk, 2012). De esta manera se sustenta el hecho de que la vasija con aplique antropomorfo haya sido parte del conjunto de utensilios empleados en la elaboración, almacenamiento y servicio de alimentos. En relación con la vasija Negro sobre Rojo (M9), no se pudo reconstruir la forma con mayor precisión, sin embargo, sabemos que constituyó una pieza más grande y de paredes más gruesas que los cuencos. Teniendo en cuenta los residuos hallados y sus características morfológicas, se podría pensar que se utilizó para almacenar, trasvasar o servir líquidos o comidas elaborados con animales rumiantes.
Es necesario mencionar que para contenedores arqueológicos pintados de otros sitios de Catamarca de los períodos Tardío e Inca se ha propuesto el posible empleo de grasas de camélidos para el sellado de las superficies internas de manera previa al almacenamiento de líquidos (Miyano et al., 2017; Lantos et al., 2020). Por esto, no se descarta que el hallazgo de grasas de animales rumiantes en las piezas pintadas y sin marcas de exposición al fuego se deba al empleo de dicha práctica.

Con respecto al análisis de la olla etnográfica, consideramos que fue fructífero, ya que colaboró con la identificación de los FAMEs en las muestras arqueológicas y nos permitió reflexionar sobre la degradación y preservación de ciertos ácidos grasos a lo largo del tiempo. El relato sobre algunos aspectos de la biografía de la vasija junto con los resultados alcanzados en este trabajo nos brindan herramientas que enriquecen las interpretaciones químicas sobre los usos de las vasijas en Loma l'Ántigo.

Sabemos que la olla etnográfica fue usada por su dueña para elaborar maíz tostado. Esto coincide con resultados preliminares inéditos del análisis de microrrestos botánicos en una parte del raspado de la vasija. Se encontró una diversidad de granos de almidón de distintas plantas, algunos de los cuales poseen marcas de degradación y/o procesamiento. Estos datos nos hablan sobre el empleo de la vasija en la elaboración de comidas que involucraron la circulación de distintas plantas.

Por otra parte, si bien no tenemos datos sobre el curado de esta olla específicamente, otra pobladora de la zona le contó a una de las autoras (Bugliani) un complejo proceso de curado realizado en sucesivos pasos, que involucra el untado con grasa, el hervido de leche y el frotado de hígado.

Consideramos que esta olla pudo haber sido curada con alguno o todos estos productos, debido a la importante presencia de ácidos grasos de origen animal, a la particular presencia de compuestos biomarcadores de rumiante y a los valores de los índices P/E e I/P. El análisis de esta olla etnográfica nos permite evaluar el alcance del uso de los índices para detectar la presencia de lípidos de origen vegetal, en el contexto particular del valle del Cajón. Las plantas poseen bajas concentraciones lipídicas en relación con los animales y, a su vez, presentan una abundancia relativa de ácidos grasos insaturados de cadena larga, que se caracterizan por sufrir mayor degradación que los ácidos grasos saturados. Es así como los resultados químicos y la 
información contextual de la vasija etnográfica nos sirven de referencia para evaluar el decrecimiento de la concentración de residuos vegetales a lo largo del tiempo y verificar el corto lapso necesario para que queden invisibilizados en el perfil de FAMEs. Por otra parte, estos índices han resultado útiles para diferenciar la presencia de lípidos de origen rumiante. Particularmente, es interesante que la mayor parte de las muestras arqueológicas presentaron índices muy similares a los de la olla etnográfica. Los dos casos que se alejaron del grupo estuvieron relacionados con el nivel de degradación, en un caso (M4), y con el aporte inusual de lípidos de origen microbiano, en el otro (M7).

Por otra parte, se destaca el hallazgo de ácidos grasos dicarboxílicos en la olla, con una alta proporción de azelaico. Como fue mencionado, se podrían asociar con la presencia de plantas. En este sentido, consideramos que tales elementos podrían comportarse como indicios de la presencia y/o degradación de lípidos de plantas, tanto en la muestra etnográfica como en las arqueológicas.

Como ocurre con la olla de referencia etnográfica, en las muestras arqueológicas, los valores del índice P/E se ubicaron, en su mayoría, en el rango de grasas de origen animal (Figura 5). El cuenco NMV8 (M7) es el único ejemplar que brindó un valor de índice $\mathrm{P} / \mathrm{E}$ que se puede interpretar como producto de una mezcla de lípidos de origen vegetal con algún aporte menor de origen animal. De acuerdo con estos valores y considerando que en todas las muestras donde se hallaron lípidos se identificaron ácidos grasos dicarboxílicos, consideramos que existe la posibilidad de que los residuos de aceites vegetales se encuentren subrepresentados en las muestras arqueológicas de Loma l'Ántigo y sea necesario realizar otros análisis que nos permitan corroborar esta suposición. Es así como se prevé analizar las fracciones de compuestos neutros de las muestras por CG-MS para indagar sobre la presencia/ausencia de distintos esteroles y/o alcoholes de cadena larga que nos puedan brindar información más específica sobre el origen de los lípidos recuperados.

\section{Agradecimientos}

Agradecemos a Dora Chaile por compartir con nosotras sus conocimientos culinarios, recuerdos e historias asociadas con el uso de la olla cerámica estudiada en este trabajo. También agradecemos a
Luis Coll por la confección del mapa de la Figura 1. Además, esta investigación pudo llevarse a cabo gracias al apoyo de la Agencia Nacional de Promoción de la Investigación, el Desarrollo Tecnológico y la Innovación (PICT-2016-0480 Dir. Lantos), CONICET (PIP-11220130100288CO Dir. Maier y PUE 20170002 Dir. Williams) y la Universidad de Buenos Aires (20020170100340BA Dir. Maier).

\section{REFERENCIAS CITADAS}

Amuedo, C. (2012). Las conexiones entre las prácticas mortuorias de infantes y los alimentos. La materialidad y los significados generados en el movimiento cotidiano. En M. P. Babot, M. Marschoff y F. Pazzarelli (Eds.), Las manos en la masa. Arqueologías, antropologías e historias de la alimentación en Suramérica (pp. 667-692). Universidad Nacional de Córdoba.

Arthur, J. W. (2002). Pottery use-alteration as an indicator of socioeconomic status. An ethnoarchaeological study of the Gamo of Ethiopia. Journal of Archaeological Method and Theory, 9(4), 331-355.

Belotti, López de Medina, C. R. y Bugliani, M. F. (2019). Primeros resultados zooarqueológicos del sitio tardío Loma l'Ántigo, valle del Cajón (Catamarca). V Congreso Nacional de Zooarqueología Argentina, San Fernando del valle de Catamarca, Argentina.

Bugliani, M. F. (2018). Evidencias del Tardío en el valle del Cajón (Catamarca): una mirada desde el sitio Loma l'Ántigo. Relaciones de la Sociedad Argentina de Antropología, XLIII(1), 35-54.

Colombini, M. P. y Modugno, F. (2009). Organic Mass Spectrometry in Art and Archaeology. Wiley.

Dudd, S. N., Regert, M. y Evershed, R. P. (1998). Assessing microbial lipid contributions during laboratory degradations of fats and oils and pure triacylglycerols absorbed in ceramic potsherds. Organic Geochemistry, 29(5-7), 1345-1354.

Dunne, J., Mercuri, A. M., Evershed, R. P., Bruni, S. y di Lernia, S. (2016). Earliest direct evidence of plant processing in prehistoric Saharan pottery. Nature plants, $3(1), 1-6$.

Eerkens, J. W. (2005). GC-MS analysis and fatty acid ratios of archaeological potsherds from the Western Great Basin of North America. Archaeometry, 47(1), 83-102.

Eerkens, J. W. (2007). Organic residue analysis and the decomposition of fatty acids in ancient potsherds. En H. Barnard y J. W. Eerkens (Eds.), Theory and Practice in Archaeological residue analysis (pp. 90-98). BAR International Series 1650. Archaeopress. 
Evershed, R. P. (2008a). Organic residue analysis in archaeology: the archaeological biomarker revolution. Archaeometry, 50(6), 895-924.

Evershed, R. P. (2008b). Experimental approaches to the interpretation of absorbed organic residues in archaeological ceramics. World Archaeology, 40(1), 26-47.

Folch, J., Lees, M. y Sloane Stanley, G. (1959). A simple method for the isolation and the purification of total lipids from animal tissues. Journal of Biological Chemistry, 226, 497-509.

Frère, M. M., Constenla, D., Bayón, C. y González, M. I. (2010). Estudios actualísticos sobre recursos silvestres mediante el empleo de análisis químicos. En M. Berón, L. Luna, M. Bonomo, C. Montalvo, C. Aranda y M. Carrera Aizpitarte (Eds.), Mamül Mapu: pasado y presente desde la arqueología pampeana (pp. 65-75). Libros del Espinillo.

Greco, C., Marchegiani, M., y Palamarczuk, V. (2012). Tipologías estilísticas e inferencias funcionales de objetos cerámicos en momentos tardíos del Noroeste Argentino. En M. P. Babot, M. Marschoff y F. Pazzarelli (Eds.), Las manos en la masa. Arqueologías, antropologías e historias de la alimentación en Suramérica (pp. 505-526). Universidad Nacional de Córdoba.

Kimpe, K., Drybooms, C., Schrevens, E., Jacobs, P. A., Degeest, R. y Waelkens, M. (2004). Assessing the relationship between form and use of different kinds of pottery from the archaeological site Sagalassos (southwest Turkey) with lipid analysis. Journal of Archaeological Science, 31(11), 1503-1510.

Lantos, I., Maier, M. S. y Ratto, N. (2012). Recreando recetas: primeros resultados de una experimentación con variedades nativas de maíz del noroeste argentino. En M. P. Babot, M. Marschoff y F. Pazzarelli (Eds.), Las manos en la masa. Arqueologías, antropologías e historias de la alimentación en Suramérica (pp. 527-552). Universidad Nacional de Córdoba.

Lantos, I., Spangenberg, J. E., Giovannetti, M. A., Ratto, N. y Maier, M. S. (2015). Maize consumption in pre-Hispanic south-central Andes: chemical and microscopic evidence from organic residues in archaeological pottery from western Tinogasta (Catamarca, Argentina). Journal of Archaeological Science, 55, 83-99.

Lantos I., Palamarczuk, V., Orgaz, M., Ratto, N. y Maier, M. S. (2018). Exploring the culinary uses of Santa María and Belén painted vessels from the Late Intermediate Period in Catamarca, Argentina. Journal of Archaeological Science: Reports, 18, 660-667.
Lantos, I., Careaga, V. P., Palamarczuk, V., Aversente, Y., Bonifazi, E., Petrucci, N. S. y Maier, M. S. (2020). Combined use of gas chromatography and HPLC-ESIQ-TOF to assess the culinary uses of archaeological Santa María style ceramic vessels from El Colorado (Catamarca, Argentina). Archaeological and Anthropological Sciences, 12, 121.

Maier, M. S., de Faria, D. L. A., Boschín, M. T., Parera, S. D. y del Castillo Bernal, M. F. (2007). Combined use of vibrational spectroscopy and GCMS methods in the characterization of archaeological pastes from Patagonia. Vibrational Spectroscopy, 44(1), 182-186.

Malainey, M. E. (1997). The reconstruction and testing of subsistence and settlement strategies for the Plains, Parkland, and Southern Boreal Forest (tesis doctoral). Departamento de Antropología, Universidad de Manitoba, Canadá.

Malainey, M. E., Przybylski, R., y Sherriff, B. L. (1999). The effects of thermal and oxidative degradation on the fatty acid composition of food plants and animals of Western Canada: implications for the identification of archaeological vessel residues. Journal of Archaeological Science, 26(1), 95-103.

Martínez Marín, A., Hernández Pérez, M., Pérez Alba, L., Gómez Castro, G., y Carrión Pardo, D. (2010). Metabolismo de los lípidos en los rumiantes. Revista Electrónica de Veterinaria, 11(8), 1-21.

Miyano, J. P., Lantos, I., Ratto, N. y Orgaz, M. (2017). Animales e Incas en el oeste Tinogasteño (Catamarca, Argentina). Latin American Antiquity, 28, 28-45.

Nastri, J. (2008). La figura de las largas cejas de la iconografía santamariana. Chamanismo, sacrificio y cosmovisión calchaquí. Boletín del Museo Chileno de Arte Precolombino, 13(1), 9-34.

Ortiz, M. G., y Heit, C. I. (2013). Nuevos avances en relación con las prácticas económicas de los grupos pedemontanos de la cuenca del San Francisco (Noroeste de Argentina, 800 AC-500 DC) a través de marcadores biomoleculares y microrrestos vegetales. Revista Española de Antropología Americana, 43(2), 369-384.

Pazzarelli, F. (2011). Una aproximación a la transformación de recursos en Piedras Blancas (Ambato, Catamarca, $\mathrm{S}$ X-XI DC) desde los análisis químicos. Relaciones de la Sociedad Argentina de Antropología, XXXVI, 331-336.

Regert, M. (2011). Analytical strategies for discriminating archaeological fatty substances from animal origin. Mass Spectrometry Reviews, 30(2), 77-220. 
Viaje al centro de la matriz cerámica: estudio sobre los usos de vasijas arqueológicas proven... Intersecciones en Antropología 22(1), enero-junio. 2021. ISSN-e 1850-373X

Roffet-Salque, M., Dunne, J., Altoft, D., Casanova, E., Cramp, L., Smyth, J., Whelton, H. J. y Evershed, R. P. (2017). From the inside out: upscaling organic residue analyses of archaeological ceramics. Journal of Archaeological Science. Report, 15, 627-640.

Sánchez Vizacíno, A., y Cañabate Guerrero, M. L. (1998). Indicadores químicos para la arqueología. Universidad de Jaén.

Skibo, J. M. (2015). Pottery use-alteration analysis in usewear and residue analysis. En J. M. Marreiros., J. F.
G. Bao y N. F. Bicho (Eds.), Use-Wear and Residue Analysis in Archaeology (pp. 189-198). Springer.

Vázquez, C., Maier, M. S., Parera, S. D., Yacobaccio, H. y Solá, P. (2008). Combining TXRF, FT-IR and GCMS information for identification of inorganic and organic components in black pigments of rock art from Alero Hornillos 2 (Jujuy, Argentina). Analytical and Bioanalytical Chemistry, 391(4), 1381-1387. 
54 | S. Fernández S. et al. - Intersecciones en Antropología 22(1), enero-junio. 2021. ISSN-e 1850-373X 